\title{
Modern Approach to Research by DSC and its Advancements
}

\section{Ashish Chauhan* and Bharti Mittu}

National Institute of Pharmaceutical Education and Research, Mohali, Punjab, India

DSC (Differential Scanning Calorimetry) is an analytical instrument that measures the heat radiated or absorbed excessively by the sample on the basis of a temperature difference between the sample and the reference material The technique was developed by E.S. Watson and M.J. O'Neill in 1962. It was introduced commercially at the 1963 Pittsburgh Conference on Analytical Chemistry and Applied Spectroscopy. The term DSC was coined to describe the instrument which measures energy directly and allows precise measurements of heat capacity and heat flux.

DSC is a magnificent technique to achieve the thermoanalytical parameters of biomolecules, nanomaterials or any other moiety. Recent advances in technology have made it possible to assess the purification yield of some pharmaceutical drugs such as antibodies. It could be useful to provide new data about the structural thermodynamics of reaction intermediates such as isothermal amplification of nucleic acids, may be processed in DSC and used to obtain new, thermodynamic information about nucleic acids while the molecules are polymerizing.

The technology can predict stability of biomolecules in various environmental conditions such as $\mathrm{pH}$, ionic strength where as some instruments can reveal volumetric features of the sample in different pressures. The interaction of biomolecules and nanoparticles could be tracked by calorimeters. Various types of calorimeters have been developed, and their applications could improve the thermoanalysis of a wide range of materials. On the other hand, in the last decades, considerable progress has been made in applications of DSCs in microfluidics, drug discovery, pharmaceuticals, molecular biology and nanoscience.

\section{Advancements in DSC}

\section{MEMS-DSC}

MEMS is a method that has been applied in the manufacturing of three-dimensional silicon-based structures with specific geometrical, mechanical and electrical characteristics to operate certain functions. The idea of obtain MEMS-DSCs was the result of two functional problems of conventional DSCs, that prevent them from performing effectively in biomolecular and structural transitions. MEMS-DSC is polymer-based miniaturized DSC with integrated microfluidics for analyzing structural transitions of biological molecules in liquid phase. MEMS-DSC contains all of the essential components, including a heating resistor, a temperature sensor, a sensor to determine temperature differences, a well-defined thermal conductance and a container. It is very effective in functioning.

\section{IR-heated DSC}

IR-heated DSC is a heat-flux DSC, that comprises of a DSC sensor assembly for receiving a sample that is installed in a cavity within an elongated cylinder and an IR lamp assembly that is disposed of circumferentially around the elongated cylinder with a length approximately similar to that of the cylinder. It is an efficient technique.

\section{MTDSC}

MTDSC provides the amplitude and phase signals i.e. alternating current $(\mathrm{AC})$ signals and the total heat-flow signal equivalent to that given by DSC simultaneously in a single experiment. The technique used a conventional DSC and the signals were obtained by deconvolution carried out by a computer. A sinusoidal temperature modulation is the program mostly used however, there are many different forms of temperature programs. It is highly advanced and fast technique.

\section{GFMDSC}

GFMDSC modulates a DSC by setting the properties of a gas in thermal contact with the sample and the reference in the calorimeter. The device allows the use of MDSC at high modulation rates as compared with the modulation rates used with MTDSC. It has high accuracy and is efficient.

\section{PNDSC}

NDSC is a power-compensation DSC that included a plurality of cell structures being used to define a selective region for calorimetric measurements of a nanomaterial. It is an efficient techniques that provides reproducible results.

The combination of calorimetric techniques available to measure the thermodynamics of folding and unfolding transitions includes differential thermal scanning (DSC), pressure scanning (PPC), and isothermal (ITC) methods. These advanced analytical instruments measure thermodynamic parameters, largely depending on different temperature and pressure ranges. These measurements may correspond to different sets of thermodynamic end states. They elaborate the properties of the solid state by characterization thus elaborating its behavior and help in comparison of measured parameters from different samples. These techniques provide modern approach and help to achieve fruitful results in research.

${ }^{*}$ Corresponding author: Ashish Chauhan, National Institute of Pharmaceutical Education and Research, Mohali, Punjab, India, E-mail: aashishchauhan26@gmail.com Received October 09, 2012; Accepted October 11, 2012; Published October 13 2012

Citation: Chauhan A, Mittu B (2012) Modern Approach to Research by DSC and its Advancements. Pharmaceut Anal Acta 3:e129. doi:10.4172/2153-2435.1000e129

Copyright: (C) 2012 Chauhan A, et al. This is an open-access article distributed under the terms of the Creative Commons Attribution License, which permits unrestricted use, distribution, and reproduction in any medium, provided the original author and source are credited. 